\title{
Reinterpretation and Reheating of (SUSY) Starobinsky model
}

\section{Takahiro TERADA*}

The University of Tokyo, DESY

E-mail: takahiro@hep-th.phys.s.u-tokyo.ac.jp

Starobinsky model of inflation is favored by observations, and it is worth searching for convincing origins of the model's specific action and possible underlying frameworks. In the first half of this presentation, we propose an explanation of the form of the action using extra dimensions based on Ref. [1], which has observational implications. In the latter half, we consider a supersymmetric embedding of the model, and study reheating processes after inflation obtaining constraints on supersymmetry breaking parameters, based on Ref. [2].

XXVII International Symposium on Lepton Photon Interactions at High Energies

17-22 August 2015

Ljubljana, Slovenia

${ }^{*}$ Speaker. 


\section{Introduction and Summary}

Starobinsky model of inflation [3] is now one of the most popular inflationary models. This is e.g. because of its simplicity and its consistency with observations. The model is characterized by a pure gravitational action which has the Einstein-Hilbert term $R$ and the square of Ricci scalar curvature $R^{2}{ }^{1}$ In contrast to many other models in which a scalar inflaton is introduced as a matter field, the inflaton emerges from an additional degree of freedom in the gravitational sector. Although it can be reformulated as Einstein gravity plus a scalar field [5], the interactions of the inflaton with matter fields are determined by its gravitational nature. This simple extension of the action of General Relativity leads to inflation compatible with the latest observation by Planck, BICEP2, and Keck-Array [6, 7].

However, the action of the Starobinsky model looks too simple. Gravity is perturbatively non-renormalizable, so higher powers of curvature invariants are generally expected. These terms must be suppressed enough for the Starobinsky model to work, while the coefficient of the $R^{2}$ term is very large. The coefficient is identified with the ratio $\left(M_{\mathrm{P}}^{2} / 12 \mathrm{~m}^{2}\right)$ where $M_{\mathrm{P}}$ is the reduced Planck mass and $m$ is the inflaton mass, which is fixed to $m \simeq 3 \times 10^{13} \mathrm{GeV}$ by the normalization of CMB fluctuation. This means that the dimensionless coefficient is about $5 \times 10^{8}$ (the precise number depends on the number of $e$-folding). It is important to study the origin of this huge coefficient and suppression of higher order terms. This is the subject of Section 2. We propose an interpretation of the form of the action based on extra dimensions [1]. The huge $R^{2}$ term is obtained from volume of extra dimensions, whereas all of the higher order terms are suppressed by one parameter tuning of order $10^{-4}$, which is technically natural in the four dimensional perspective.

It is useful to study not only theoretical aspects as above but also phenomenology during or after inflation. In comparing observational data with theoretical predictions, we are interested in distinguishing various inflationary models. There are, however, many models whose predictions are (almost) the same as those of the Starobinsky model. It is then interesting to consider physics after inflation for more detailed comparisons of those Starobinsky-like models and for constraints on the parameter space of each of the models. For example, Starobinsky inflation and Higgs inflation [8] can be distinguished by their reheating temperatures [9]. As one of the steps toward this goal, in Section 3, we focus on the supersymmetric (SUSY) Starobinsky model in the old-minimal supergravity [10,11] based on Ref. [2]. The reheating temperature is similar to the non-SUSY case although the main decay channel tends to be different. We estimate abundance of gravitino or lightest SUSY particle (LSP) from inflaton decay, and obtain constraints on masses of gravitino and the SUSY breaking field. More detailed discussions can be found in Refs. [1, 2].

\section{Reinterpretation of Starobinsky model}

We explain the large coefficient of the $R^{2}$ term (or equivalently the small inflaton mass compared to the Planck scale) by a volume factor of extra dimensions. Let us consider the following

\footnotetext{
${ }^{1}$ The original version includes other (tensor) quadratic curvature terms, but these are irrelevant and the general form is described by the scalar curvature in the conformally flat background. In this contribution, we refer the model of the form $R+\alpha R^{2}$ with $\alpha$ constant [4] to the Starobinsky model as is common in the literature.
} 
$D$-dimensional gravitational action,

$$
S=\int \mathrm{d}^{D} x \sqrt{-g_{D}} \Lambda^{D} \sum_{n=0}^{\infty} b_{n}\left(\frac{R_{D}}{\Lambda^{2}}\right)^{n},
$$

which is characterized by a typical energy scale $\Lambda . g_{D}$ and $R_{D}$ are the $D$-dimensional determinant of metric and Ricci scalar, and $b_{n}$ 's are constants generically of order one. We take $b_{2}=1$ by field redefinition of the metric.

Suppose e.g. $D=10$ and that the extra dimensions are compactified to a length of order $L \simeq 30 / \Lambda$. Then, the effective four-dimensional action is $S=\int \mathrm{d}^{4} x \sqrt{-g} c \Lambda^{4} \sum_{n=0}^{\infty} b_{n}\left(R / \Lambda^{2}\right)^{n}$, where the overall dimensionless constant is given by $c=\Lambda^{6} L^{6} \simeq 5 \times 10^{8}$.

After fixing $b_{n}(n=0,1,2)$ so that it reproduces the terms of the Starobinsky model, $\Lambda$ can be expressed in terms of $M_{\mathrm{P}}, m$, and $b_{1}(<0)$. Since all the higher order terms are scaled by powers of $\Lambda$, they are now controlled only by $b_{1}$,

$$
S=S_{\text {Starobinsky }}+\int \mathrm{d}^{4} x \sqrt{-g} \frac{M_{\mathrm{P}}^{2}}{12 m^{2}} \sum_{n=3}^{\infty} b_{n}\left(\frac{\left|b_{1}\right|}{6 m^{2}}\right)^{n-2} R^{n} .
$$

Thus, if we tune one parameter $\left|b_{1}\right|$ to be small, all the higher terms are suppressed with order one $b_{n}$. From consistency with observations, $\left|b_{1}\right| \lesssim 10^{-4}$ is required.

\section{Reheating processes of SUSY Starobinsky model}

The Starobinsky model is equivalent to the Einstein action plus a real scalar field (inflaton) with its specific potential. The analogue holds for the SUSY version [10]. We study the standard supergravity coupled to two chiral superfields $T$ (inflaton) and $S$ (stabilizer) which is equivalent to the SUSY Starobinsky model. We allow generic dependence of the action on the super-curvature, but assume the minimal coupling between (super)gravity and matter in the Jordan frame. To discuss gravitino production, we also consider the SUSY breaking sector. For definiteness, we take the Polonyi model stabilized strongly by the quartic term in the Kähler potential. The Polonyi field is denoted by $Z$. Respecting the philosophy of the original Starobinsky model, we assume the minimal mediation of SUSY breaking, i.e. anomaly mediation. (For gravitino lighter than about $30 \mathrm{TeV}$, we assume gravity mediation to avoid too light gauginos.)

The dominant decay mode is somewhat model dependent. If there is a scalar particle whose mass is slightly smaller than the half of the inflaton mass, the inflaton decays mainly into the scalar. An example is the right-handed sneutrino. If there is a sizable holomorphic bilinear term in the Kähler potential for some field (e.g. Higgs fields with the Giudice-Masiero mechanism), the inflaton decays mainly into the field. As a less model-dependent channel, there is super-Weyl-Kähler and sigma-model anomaly-induced decay into gauge bosons or gauginos. We assume this is the dominant channel. The reheating temperature is found to be $T_{\mathrm{R}} \simeq 1 \times 10^{9} \mathrm{GeV}$, a similar value to the non-SUSY case.

The abundances of gravitino and the LSP are constrained by the big-bang nucleosynthesis (BBN) and dark matter abundance. We consider four production mechanisms of gravitino: (i) direct production, (ii) thermal production, (iii) Polonyi particle decay, and (iv) Polonyi field decay. The LSP is produced thermally, or non-thermally from gravitino. The constraint is shown in Fig. 
1 of Ref. [2]. The bottom white region is also constrained by the cosmological moduli problem, so the only the middle white region is allowed. The masses of gravitino and the SUSY breaking field are constrained as $10^{4} \mathrm{GeV} \lesssim m_{3 / 2} \lesssim 10^{5} \mathrm{GeV}$ and $10^{8.5} \mathrm{GeV} \lesssim m_{Z} \lesssim 10^{9.5} \mathrm{GeV}$, respectively. Some ways around this are $R$-parity violation, thermal inflation, or non-minimal coupling between (super)gravity and matter.

\section{Acknowledgement}

The author is grateful to the collaborators of the papers this contribution is based on: Takehiko Asaka, Satoshi Iso, Hikaru Kawai, Kazunori Kohri, and Toshifumi Noumi for Ref. [1], and Yuki Watanabe, Yusuke Yamada, and Jun'ichi Yokoyama for Ref. [2]. The author's works are supported by a Grant-in-Aid for JSPS Fellows and the Grant-in-Aid for Scientific Research No. 26•10619.

\section{References}

[1] T. Asaka, S. Iso, H. Kawai, K. Kohri, T. Noumi and T. Terada, "Reinterpretation of the Starobinsky model," arXiv:1507.04344 [hep-th].

[2] T. Terada, Y. Watanabe, Y. Yamada and J. Yokoyama, "Reheating processes after Starobinsky inflation in old-minimal supergravity,” JHEP 1502 (2015) 105 [arXiv:1411.6746 [hep-ph]].

[3] A. A. Starobinsky, "A New Type of Isotropic Cosmological Models Without Singularity,” Phys. Lett. B 91 (1980) 99; in: Quantum Gravity, Proc. of the Second Seminar "Quantum Theory of Gravity" (Moscow, 13-15 Oct. 1981), eds. M. A. Markov and P. C. West (Plenum Publ. Co., New York, 1984) pp. 103-128.

[4] M. B. Mijic, M. S. Morris and W. -M. Suen, "The $R^{* * 2}$ Cosmology: Inflation Without a Phase Transition,” Phys. Rev. D 34, 2934 (1986).

[5] B. Whitt, "Fourth Order Gravity as General Relativity Plus Matter," Phys. Lett. B 145 (1984) 176;

K. Maeda, "Inflation as a Transient Attractor in $R^{* * 2}$ Cosmology," Phys. Rev. D 37, 858 (1988).

J. D. Barrow, “The Premature Recollapse Problem in Closed Inflationary Universes,” Nucl. Phys. B 296 (1988) 697.

[6] P. A. R. Ade et al. [BICEP2 and Planck Collaborations], "Joint Analysis of BICEP2/KeckArray and Planck Data,” Phys. Rev. Lett. 114 (2015) 10, 101301 [arXiv:1502.00612 [astro-ph.CO]].

[7] P. A. R. Ade et al. [Planck Collaboration], "Planck 2015 results. XX. Constraints on inflation," arXiv:1502.02114 [astro-ph.CO].

[8] F. L. Bezrukov and M. Shaposhnikov, "The Standard Model Higgs boson as the inflaton," Phys. Lett. B 659 (2008) 703 [arXiv:0710.3755 [hep-th]].

[9] F. L. Bezrukov and D. S. Gorbunov, "Distinguishing between $R^{2}$-inflation and Higgs-inflation," Phys. Lett. B 713 (2014) 365 [arXiv:1111.4397 [hep-ph]].

[10] S. Cecotti, "Higher Derivative Supergravity Is Equivalent To Standard Supergravity Coupled To Matter. 1.," Phys. Lett. B 190 (1987) 86.

[11] R. Kallosh and A. Linde, "Superconformal generalizations of the Starobinsky model," JCAP 1306 (2013) 028 [arXiv:1306.3214 [hep-th]]. 